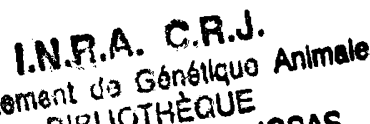 \\ EIQLLOTHECUE \\ F.78352 JOUY EN JOSAS
}

\author{
Original article
}

\title{
Gibbs sampling, adaptive rejection sampling and robustness to prior specification for a mixed linear model
}

\author{
CM Theobald, MZ Firat*, R Thompson ${ }^{\dagger}$ \\ Department of Mathematics and Statistics, University of Edinburgh, \\ King's Buildings, Mayfield Road, Edinburgh EH9 3JZ, UK
}

(Received 4 October 1995; accepted 13 January 1997)

\begin{abstract}
Summary - Markov chain Monte-Carlo methods are increasingly being applied to make inferences about the marginal posterior distributions of parameters in quantitative genetic models. This paper considers the application of one such method, Gibbs sampling, to Bayesian inferences about parameters in a normal mixed linear model when a restriction is imposed on the relative values of the variance components. Two prior distributions are proposed which incorporate this restriction. For one of them, the adaptive rejection sampling technique is used to sample from the conditional posterior distribution of a variance ratio. Simulated data from a balanced sire model are used to compare different implementations of the Gibbs sampler and also inferences based on the two prior distributions. Marginal posterior distributions of the parameters are illustrated. Numerical results suggest that it is not necessary to discard any iterates, and that similar inferences are made using the two prior specifications.
\end{abstract}

adaptive rejection sampling / Bayesian inference / Gibbs sampling / mixed linear model / sire model

Résumé - Échantillonnage de Gibbs, échantillonnage de rejet adapté et robustesse relative à l'a priori spécifié pour un modèle linéaire mixte. On utilise de plus en plus des méthodes de Monte-Carlo basées sur des chaînes de Markov pour faire des inférences sur des distributions marginales a posteriori des paramètres de modèles génétiques quantitatifs. Cet article concerne l'application d'une telle méthode, l'échantillonnage de Gibbs, à des inférences bayésiennes sur des paramètres d'un modèle linéaire mixte normal, quand on impose une contrainte sur les valeurs relatives des composantes de variance. Deux distributions a priori incorporant cette contrainte sont proposées. Pour l'une d'elles, la technique de l'échantillonnage de rejet adapté est employée pour échantillonner dans une distribution conditionnelle a posteriori d'un rapport de variance. Des données simulées

\footnotetext{
*Present address: Department of Animal Science, University of Çukurova, Faculty of Agriculture, 01330 Balcalı, Adana, Turkey.

${ }^{\dagger}$ Present address: Rothamsted Experimental Station, Harpenden, Hertfordshire AL5 2JQ, UK.
} 
dans un modèle père équilibré sont utilisées pour comparer différentes mises en œuvre de l'échantillonnage de Gibbs et aussi les inférences basées sur les deux distributions a priori. Les distributions marginales a posteriori des paramètres sont données comme illustrations. Les exemples numériques suggèrent qu'il n'est nécessaire d'éliminer aucun résultat d'itération et que des inférences similaires sont faites en utilisant l'un ou l'autre des deux a priori.

échantillonnage de rejet adapté / inférence bayésienne / échantillonnage de Gibbs / modèle linéaire mixte / modèle père

\section{INTRODUCTION}

Parameters such as heritability, and predictors of individual breeding values, depend on the unknown variance components in quantitative genetic models. The wide array of methods available for estimating variance components includes ANOVA, likelihood-based methods and Bayesian methods. A difficulty with ANOVA methods which has concerned many authors is that ANOVA estimates may lie outside the parameter space, for example estimates of heritabilities which are negative or greater than one. The use of such estimates for genetic parameters can lead to very inefficient selection decisions.

Owing to rapid advances in computing technology, methods based on likelihood have become common in animal breeding; in particular REML (Patterson and Thompson, 1971) has been widely used. REML estimators are by definition always within the parameter space, and they are consistent, asymptotically normal and efficient (Harville, 1977). However, interval estimates for variances based on the asymptotic distribution of REML estimators can include negative values (Gianola and Foulley, 1990).

In recent years, Bayesian methods have been applied to variance component estimation in animal breeding (Harville, 1977; Gianola and Fernando, 1986; Gianola et al, 1986; Foulley et al, 1987; Cantet et al, 1992; Wang et al, 1993, 1994). For normal models, the joint posterior distribution of the dispersion parameters can be derived, but numerical integration techniques are required to obtain the marginal distributions of functions of interest.

Gibbs sampling is a Markov chain Monte-Carlo method which operates by generating samples from a sequence of conditional distributions; under suitable conditions, its equilibrium distribution is the joint posterior distribution. Gilks et al (1996) provide a thorough introduction to the Gibbs sampler. The method is applied to variance component estimation in a general univariate mixed linear model by Wang et al (1993). Also, Wang et al (1994) obtain marginal inferences about fixed and random effects, variance components and functions of them through a scalar version of the Gibbs sampling algorithm.

The objective of this paper is to broaden the application of Gibbs sampling in an animal breeding context by considering prior distributions which incorporate a constraint on the relative values of variance components: such a constraint might arise from restricting heritability to values less than one. The next section defines the model to be considered and sets out two possible forms for the prior distribution: the second of these is in terms of one variance and a variance ratio. The following section describes how the techniques of Gibbs sampling and adaptive rejection 
sampling can be applied to inferences about model parameters under the two specifications. Simulated data from a balanced univariate one-way sire model are used to demonstrate these methods, to compare three implementations of Gibbs sampling and to examine the robustness of the inferences to the choice of prior.

\section{THE MODEL AND ALTERNATIVE PRIOR DISTRIBUTIONS}

\section{Model}

We consider inferences about model parameters for a univariate mixed linear model of the form

$$
\mathbf{y}=\mathbf{X} \boldsymbol{\beta}+\mathbf{Z u}+\mathbf{e}
$$

where $\mathbf{y}$ and $\mathbf{e}$ are $n$-vectors representing observations and residual errors, $\boldsymbol{\beta}$ is a $p$-vector of 'fixed effects', $\mathbf{u}$ is a $q$-vector of 'random effects', $\mathbf{X}$ is a known $n \times p$ matrix of full column rank and $\mathbf{Z}$ is a known $n \times q$ matrix. Here $\mathbf{e}$ is assumed to have the distribution $N_{n}\left(\mathbf{0}, \sigma_{e}^{2} \mathbf{I}\right)$, independently of $\boldsymbol{\beta}$ and $\mathbf{u}$. Also $\mathbf{u}$ is taken to be $N_{q}\left(\mathbf{0}, \sigma_{u}^{2} \mathbf{G}\right)$ with $\mathbf{G}$ a known positive definite matrix.

\section{Restrictions on variance ratios}

In animal breeding applications, interest may be in making inferences about ratios of variances or functions thereof, rather than about individual variance components themselves. For example, if $\mathbf{u}$ and $\mathbf{y}$ represent vectors of sire effects and of phenotypic values recorded on the sires' offspring in unrelated paternal half-sib families, and $\gamma$ denotes the variance ratio $\sigma_{u}^{2} / \sigma_{e}^{2}$, then the heritability, $h^{2}$, of the trait is an increasing function of $\gamma$ given by $h^{2}=4 /\left(1+\gamma^{-1}\right)$. The constraint that the heritability is between 0 and 1 restricts $\gamma$ to lie in the interval $\left(0, \frac{1}{3}\right)$. A method of inference which ignores such restrictions may lead to ridiculous estimates of heritability. More generally, we might consider restricting $\gamma$ to an interval $(0, g)$, corresponding to the constraint that $0<\sigma_{u}^{2}<g \sigma_{e}^{2}$, where $g$ is a known constant.

\section{Bayesian formulation with variance parameters $\sigma_{e}^{2}$ and $\sigma_{u}^{2}$}

In the above model, the assumption made about the distribution of $\mathbf{u}$ must be supplemented by prior distributions for $\boldsymbol{\beta}, \sigma_{e}^{2}$ and $\sigma_{u}^{2}$. In specifying their form, we might aim to reflect the animal breeder's prior knowledge while permitting convenient calculations on the resulting posterior distributions.

The prior distribution of $\boldsymbol{\beta}$ is taken here to be uniform, corresponding to little being known about its value initially. Wang et al $(1993,1994)$ make the same prior assumption about $\boldsymbol{\beta}$ (in a model with further random effects), and consider taking $\sigma_{e}^{2}$ and $\sigma_{u}^{2}$ to be independent with scaled inverse- $\chi^{2}$ distributions $\chi^{-2}\left(\nu_{e},\left(\nu_{e} k_{e}\right)^{-1}\right)$ and $\chi^{-2}\left(\nu_{u},\left(\nu_{u} k_{u}\right)^{-1}\right) ; k_{e}^{-1}$ and $k_{u}^{-1}$ can then be interpreted as prior expectations of $\sigma_{e}^{-2}$ and $\sigma_{u}^{-2}$, respectively, and $\nu_{e}$ and $\nu_{u}$ as precision parameters analogous to degrees of freedom. 
This prior assumption may be modified to take account of an upper bound on the variance ratio, so that $\sigma_{e}^{2}$ and $\sigma_{u}^{2}$ are not independent, but have a joint prior density of the form

$$
\begin{array}{r}
f\left(\sigma_{e}^{2}, \sigma_{u}^{2} \mid \nu_{e}, k_{e}, \nu_{u}, k_{u}\right) \propto\left(\sigma_{e}^{2}\right)^{-\frac{1}{2}\left(\nu_{e}+2\right)}\left(\sigma_{u}^{2}\right)^{-\frac{1}{2}\left(\nu_{u}+2\right)} \exp \left[-\frac{1}{2}\left(\frac{\nu_{e} k_{e}}{\sigma_{e}^{2}}+\frac{\nu_{u} k_{u}}{\sigma_{u}^{2}}\right)\right] \\
\left(0<\sigma_{e}^{2}<\infty, 0<\sigma_{u}^{2}<g \sigma_{e}^{2}\right)
\end{array}
$$

The conditional density of $\mathbf{y}$ given $\boldsymbol{\beta}, \mathbf{u}$ and $\sigma_{e}^{2}$ for the model given in [1] is proportional to $\left(\sigma_{e}^{2}\right)^{-\frac{1}{2} n} \exp \left[-\frac{1}{2} \sigma_{e}^{-2}(\mathbf{y}-\mathbf{X} \boldsymbol{\beta}-\mathbf{Z u})^{\prime}(\mathbf{y}-\mathbf{X} \boldsymbol{\beta}-\mathbf{Z u})\right]$, so the joint posterior density is given by

$$
\begin{aligned}
& f_{1}\left(\boldsymbol{\beta}, \mathbf{u}, \sigma_{e}^{2}, \sigma_{u}^{2} \mid \mathbf{y}\right) \propto\left(\sigma_{e}^{2}\right)^{-\frac{1}{2}\left(n+\nu_{e}+2\right)}\left(\sigma_{u}^{2}\right)^{-\frac{1}{2}\left(q+\nu_{u}+2\right)} \\
& \quad \times \exp \left\{-\frac{1}{2 \sigma_{e}^{2}}\left[(\mathbf{y}-\mathbf{X} \boldsymbol{\beta}-\mathbf{Z u})^{\prime}(\mathbf{y}-\mathbf{X} \boldsymbol{\beta}-\mathbf{Z u})+\nu_{e} k_{e}\right]-\frac{1}{2 \sigma_{u}^{2}}\left[\mathbf{u}^{\prime} \mathbf{G}^{-1} \mathbf{u}+\nu_{u} k_{u}\right]\right\} \\
& \left(\boldsymbol{\beta} \in R^{p}, \mathbf{u} \in R^{q}, 0<\sigma_{e}^{2}<\infty, 0<\sigma_{u}^{2}<g \sigma_{e}^{2}\right)
\end{aligned}
$$

It is shown in the Appendix that if $\nu_{u}$ is positive and $n+\nu_{e}$ exceeds $p$ then the marginal posterior distribution of $\gamma$ is proper and hence this joint distribution is also.

To implement the Gibbs sampling algorithm, we require the conditional posterior distributions of each of $\beta, \mathbf{u}, \sigma_{e}^{2}$ and $\sigma_{u}^{2}$ given the remaining parameters, the socalled full conditional distributions, which are as follows (using the notation [.|.] for a conditional distribution)

$$
\begin{gathered}
{\left[\boldsymbol{\beta} \mid \mathbf{u}, \sigma_{e}^{2}, \sigma_{u}^{2}, \mathbf{y}\right]=N_{p}\left(\left(\mathbf{X}^{\prime} \mathbf{X}\right)^{-1} \mathbf{X}^{\prime}(\mathbf{y}-\mathbf{Z u}), \sigma_{e}^{2}\left(\mathbf{X}^{\prime} \mathbf{X}\right)^{-1}\right)} \\
{\left[\mathbf{u} \mid \boldsymbol{\beta}, \sigma_{e}^{2}, \sigma_{u}^{2}, \mathbf{y}\right]=N_{q}\left(\left(\mathbf{Z}^{\prime} \mathbf{Z}+\gamma^{-1} \mathbf{G}^{-1}\right)^{-1} \mathbf{Z}^{\prime}(\mathbf{y}-\mathbf{X} \boldsymbol{\beta}), \sigma_{e}^{2}\left(\mathbf{Z}^{\prime} \mathbf{Z}+\gamma^{-1} \mathbf{G}^{-1}\right)^{-1}\right)[5]} \\
{\left[\sigma_{e}^{2} \mid \boldsymbol{\beta}, \mathbf{u}, \sigma_{u}^{2}, \mathbf{y}\right]=\chi^{-2}\left(n+\nu_{e},\left[(\mathbf{y}-\mathbf{X} \boldsymbol{\beta}-\mathbf{Z u})^{\prime}(\mathbf{y}-\mathbf{X} \boldsymbol{\beta}-\mathbf{Z u})+\nu_{e} k_{e}\right]^{-1}\right)} \\
{\left[\sigma_{u}^{2} \mid \boldsymbol{\beta}, \mathbf{u}, \sigma_{e}^{2}, \mathbf{y}\right]=\chi^{-2}\left(q+\nu_{u},\left[\mathbf{u}^{\prime} \mathbf{G}^{-1} \mathbf{u}+\nu_{u} k_{u}\right]^{-1}\right)}
\end{gathered}
$$

(truncated at $g \sigma_{e}^{2}$ ). Apart from the use here of proper prior distributions for the variances, these are similar to the conditional distributions given in Wang et al (1993). Methods for sampling from these distributions are well known.

\section{An alternative Bayesian formulation with prior independence of $\sigma_{e}^{2}$ and $\gamma$}

As Wang et al (1993) show for a model with a slightly simpler prior for $\sigma_{e}^{2}$ and $\sigma_{u}^{2}$, the prior specification in terms of these two parameters is a convenient one for applying the method of Gibbs sampling. It is also easily generalized when several traits are recorded. It may not, however, be the most useful specification for eliciting the prior opinions of animal breeders about parameter values. The animal breeder 
may prefer to think in terms of the heritability of a trait, equivalent for a paternal half-sib model to the variance ratio $\gamma=\sigma_{u}^{2} / \sigma_{e}^{2}$ defined above. A second function of the two variances must also be considered in order to specify their joint prior distribution, and $\sigma_{e}^{2}$ may be an appropriate choice because inferences about $\sigma_{e}^{2}$ from previous data are likely to be much more precise than those about $\sigma_{u}^{2}$. To specify a joint prior distribution for the sire and residual variance components, it may therefore be convenient to assign priors to $\sigma_{e}^{2}$ and the variance ratio $\gamma$. We thus consider the alternative parameter vector $\left(\boldsymbol{\beta}, \mathbf{u}, \sigma_{e}^{2}, \gamma\right)$, but make the same prior assumptions for $\boldsymbol{\beta}$ and $\mathbf{u}$ as before, noting that $\sigma_{u}^{2}$ becomes $\gamma \sigma_{e}^{2}$. We can incorporate an upper bound $g$ on the range of $\gamma$ more naturally than with the previous formulation by using the family of Beta distributions on the interval $(0, g)$ : this is a fairly flexible family with probability density of the form

$$
f(\gamma \mid a, b) \propto \gamma^{a-1}(g-\gamma)^{b-1} \quad(0<\gamma<g)
$$

If $\gamma$ is taken to be independent of $\boldsymbol{\beta}$ and $\sigma_{e}$ a priori, and $\sigma_{e}^{2}$ assumed to be $\chi^{-2}\left(\nu_{e},\left(\nu_{e} k_{e}\right)^{-1}\right)$ then the joint posterior density is given by

$$
\begin{aligned}
& f_{2}\left(\boldsymbol{\beta}, \mathbf{u}, \sigma_{e}^{2}, \gamma \mid \mathbf{y}\right) \propto\left(\sigma_{e}^{2}\right)^{-\frac{1}{2}\left(n+q+\nu_{e}+2\right)} \gamma^{a-\frac{1}{2} q-1}(g-\gamma)^{b-1} \\
& \quad \times \exp \left\{-\frac{1}{2 \sigma_{e}^{2}}\left[(\mathbf{y}-\mathbf{X} \boldsymbol{\beta}-\mathbf{Z u})^{\prime}(\mathbf{y}-\mathbf{X} \boldsymbol{\beta}-\mathbf{Z} \mathbf{u})+\nu_{e} k_{e}+\gamma^{-1} \mathbf{u}^{\prime} \mathbf{G}^{-1} \mathbf{u}\right]\right\} \\
& \quad\left(\boldsymbol{\beta} \in R^{p}, \mathbf{u} \in R^{q}, 0<\sigma_{e}^{2}<\infty, 0<\gamma<g\right)
\end{aligned}
$$

The full conditional posterior distributions for $\boldsymbol{\beta}$ and $\mathbf{u}$ are the same as [4] and [5], while that for $\sigma_{e}^{2}$ is

$$
\chi^{-2}\left(n+q+\nu_{e},\left[(\mathbf{y}-\mathbf{X} \boldsymbol{\beta}-\mathbf{Z u})^{\prime}(\mathbf{y}-\mathbf{X} \boldsymbol{\beta}-\mathbf{Z} \mathbf{u})+\nu_{e} k_{e}+\gamma^{-1} \mathbf{u}^{\prime} \mathbf{G}^{-1} \mathbf{u}\right]^{-1}\right)
$$

The full conditional density for $\gamma$ is proportional to

$$
\gamma^{a-\frac{1}{2} q-1}(g-\gamma)^{b-1} \exp \left(-\frac{\mathbf{u}^{\prime} \mathbf{G}^{-1} \mathbf{u}}{2 \gamma \sigma_{e}^{2}}\right) \quad(0<\gamma<g)
$$

This family is not a well-known one, so it is not immediately clear how to sample from it. However, the adaptive rejection sampling technique of Gilks and Wild (1992) provides a reasonably efficient method for sampling from the distribution of $\ln \gamma$.

Given these two methods of including the restriction on $\sigma_{u}^{2} / \sigma_{e}^{2}$, the question arises of how posterior inferences, especially about $\gamma$, are affected when the second prior specification is used instead of the first. To answer this, we consider alternative specifications in which the priors for the variance parameters are similar: to match these distributions we choose $a$ and $b$ in [8] to give $\gamma$ the same upper and lower quartiles under both. Details are given in the Appendix. 


\section{CALCULATIONS ON THE POSTERIOR DISTRIBUTIONS}

\section{Gibbs sampling}

Markov chain Monte-Carlo methods, particularly Gibbs sampling, are now widely used for making inferences from posterior distributions of parameters. Gelfand and Smith (1990) review the Gibbs sampler and other Monte-Carlo methods, and Gelfand et al (1990) illustrate the use of the Gibbs sampler on a range of statistical models, including variance components models. Wang et al $(1993,1994)$ give two versions of the Gibbs sampler for a univariate normal mixed linear model assuming prior independence of the variance components.

The Gibbs sampler algorithm generates samples iteratively from the full conditional distributions of disjoint subsets of the parameters given the current value of the complementary subset.

The following version of the Gibbs sampler can be applied to sample from the posterior distribution defined in [3] using the full conditional distributions given in [4]-[7]. It incorporates the restriction that $\sigma_{u}^{2} / \sigma_{e}^{2}$ cannot exceed an upper bound $g$ by discarding pairs of variances which do not satisfy this condition.

(i) Choose an arbitrary starting value $\boldsymbol{\theta}^{(0)}=\left(\boldsymbol{\beta}^{(0)}, \mathbf{u}^{(0)}, \sigma_{e}^{2(0)}, \sigma_{u}^{2(0)}\right)$ for the vector of parameters $\boldsymbol{\theta}=\left(\boldsymbol{\beta}, \mathbf{u}, \sigma_{e}^{2}, \sigma_{u}^{2}\right)$;

(ii) draw a value $\boldsymbol{\beta}^{(1)}$ from the distribution $\left[\boldsymbol{\beta} \mid \mathbf{u}^{(0)}, \sigma_{e}^{2(0)}, \sigma_{u}^{2(0)}, \mathbf{y}\right]$;

(iii) draw $\mathbf{u}^{(1)}$ from $\left[\mathbf{u} \mid \boldsymbol{\beta}^{(1)}, \sigma_{e}^{2(0)}, \sigma_{u}^{2(0)}, \mathbf{y}\right]$;

(iv) draw $\sigma_{u}^{2(1)}$ from $\left[\sigma_{u}^{2} \mid \boldsymbol{\beta}^{(1)}, \mathbf{u}^{(1)}, \sigma_{e}^{2(0)}, \mathbf{y}\right]$ ignoring the restriction on $\sigma_{u}^{2} / \sigma_{e}^{2}$;

(v) $\operatorname{draw} \sigma_{e}^{2(1)}$ from $\left[\sigma_{e}^{2} \mid \boldsymbol{\beta}^{(1)}, \mathbf{u}^{(1)}, \sigma_{u}^{2(1)}, \mathbf{y}\right]$ ignoring the restriction on $\sigma_{u}^{2} / \sigma_{e}^{2}$;

(vi) if $\sigma_{u}^{2(1)} / \sigma_{e}^{2(1)} \geqslant g$ then repeat (iv) and (v) until $\sigma_{u}^{2(1)} / \sigma_{e}^{2(1)}<g$.

Thus, subsets of the parameters are sampled in an arbitrary order and this cycle defines the transition from $\boldsymbol{\theta}^{(0)}$ to $\boldsymbol{\theta}^{(1)}$. Subsequent cycles replace $\boldsymbol{\theta}^{(1)}$ to give further vectors $\boldsymbol{\theta}^{(2)}, \boldsymbol{\theta}^{(3)}$, and so on.

Opinions differ on how the sequence of vectors used in subsequent analyses should be defined. The following three methods of implementation have been proposed, and are compared on simulated data in the next section.

(a) A single long chain: one generates a single run of the chain as practised by Geman and Geman (1984), ie,

(vii) repeat (ii)-(vi) $m$ times using updated values and store all the values.

A common modification is to discard vectors from the start of the sequence to allow it to approach equilibrium. The remaining vectors $\boldsymbol{\theta}^{(1)}, \ldots, \boldsymbol{\theta}^{(m)}$ approximate simulated values from the posterior distribution of $\boldsymbol{\theta}$, but successive vectors may be correlated and the sequence may be strongly influenced by the starting values.

(b) Equally spaced samples: to reduce serial correlations, one can choose suitable integers $k$ and $m$, perform a single run of length $k m$, and then form a sample from every $k$ th vector, ie, use $\boldsymbol{\theta}^{(k)}, \boldsymbol{\theta}^{(2 k)}, \ldots, \boldsymbol{\theta}^{(k m)}$.

(c) Multiple short chains: by contrast, Gelfand and Smith (1990) and Gelfand et al (1990) perform $m$ runs each of length $l$, with different starting values, forming a sample by using the last iterate from each run. 
In support of implementation (c), Gelman and Rubin (1992) argue that, even if using one long run may be more efficient, it may still be important to use several different starting points.

It has been common practice to discard a substantial number of initial iterations, and to use implementations (b) and (c) (especially (b), storing only every $k$ th, usually 10 th or 20 th, iterate). The results of Raftery and Lewis (1992) suggest that in many cases this is rather profligate.

For any individual parameter, the collection of $m$ values can be viewed as a simulated sample from the appropriate marginal distribution. This sample can be used to calculate summaries, such as the marginal posterior mean, or to estimate marginal posterior distributions of parameters or of functions of them using kernel density estimation. We use kernel density estimation below with normal kernels and window width suggested by Silverman (1986).

\section{Adaptive rejection sampling}

The adaptive rejection sampling technique introduced by Gilks and Wild (1992) is a method of rejection sampling intended for situations in which evaluation of a univariate density is computationally expensive. It uses a squeezing function (Devroye, 1986), and is adaptive in the sense that the rejection envelope and squeezing function (which are piecewise exponential) are revised as sampling proceeds, and approach the density function. In Gibbs sampling, the full conditional distribution for each parameter changes as the iteration proceeds, so that usually only one value is required from a particular density. Adaptive rejection sampling has the advantage that it does not require an optimization step to locate the mode of the density before a sample can be generated.

The method requires that the density is log-concave, that is that the derivative of the logarithm of the density is monotone decreasing. It is shown in the Appendix that the density of $\gamma$ in expression [11] is guaranteed to be log-concave only if $a$ is greater than $\frac{1}{2} q+1$, but that if we transform to $\delta=\ln \gamma$ then the full conditional density of $\delta$ is log-concave if $b$ is at least one. The condition on $a$ is not a reasonable one, since a prior with a maximum at zero must have $a$ no greater than one, for example. However, a value of $b$ less than one implies that the prior density in [8] has a maximum at its upper bound, $g$ (which might correspond to a heritability of one), and it is not unreasonable to exclude such values. Hence we use adaptive rejection sampling with $\ln \gamma$.

\section{APPLICATION TO A ONE-WAY CLASSIFICATION}

The methods described above were applied to a one-way sire model of the form

$$
y_{i j}=\beta+u_{i}+e_{i j} \quad(i=1, \ldots, s ; j=1, \ldots, d)
$$

This is intended to represent observations of a trait on the $d$ half-sib offspring of each of $s$ sires: $\beta$ and the $u_{i}$ are the overall expectation and the $i$ th sire effect, respectively. It is a particular case of the model given in [1] 
in which $p$ and $q$ equal 1 and $s$, and $\mathbf{X}, \mathbf{Z}$ and $\mathbf{G}$ become $\mathbf{1}_{s d}$ (a vector of 1s with length $s d), \operatorname{diag}\left(\mathbf{1}_{d}, \ldots, \mathbf{1}_{d}\right)$ and $\mathbf{I}_{s}$. The full conditional distributions for $\beta, \mathbf{u}, \sigma_{e}^{2}$ and $\sigma_{u}^{2}$ defined in expressions [4]-[7] become $N\left(\bar{y}_{. .}-\bar{u}_{.}, \frac{\sigma_{e}^{2}}{s d}\right)$, $N_{s}\left(\frac{d \gamma}{d \gamma+1}\left(\overline{\mathbf{y}}_{.}-\beta \mathbf{1}_{s}\right), \frac{\gamma \sigma_{e}^{2}}{d \gamma+1} \mathbf{I}_{s}\right), \chi^{-2}\left(s d+\nu_{e},\left[\sum_{i} \sum_{j}\left(y_{i j}-\beta-u_{i}\right)^{2}+\nu_{e} k_{e}\right]^{-1}\right)$ and $\chi^{-2}\left(s+\nu_{u},\left[\sum_{i} u_{i}^{2}+\nu_{u} k_{u}\right]^{-1}\right)$, where $\overline{\mathbf{y}}_{.}, \bar{y}_{\text {.. }}$ and $\bar{u}$. denote the vector of family means and the overall means of the $y_{i j}$ and the $u_{i}$.

Four sets of data were generated using this model with parameter values $\beta=0$, $\sigma_{e}^{2}=0.975, \sigma_{u}^{2}=0.025$ and hence $h^{2}=0.1$ with 25 families and 20 offspring per sire. ANOVA estimates are summarized in table I: the data sets are ordered by the estimates of heritability, which range from -0.073 to 0.306 . The first ANOVA estimate of $\sigma_{u}^{2}$ is negative, making conventional inference about this parameter difficult.

Table I. ANOVA estimates for the four data sets.

\begin{tabular}{lrrrrr}
\hline Data sets & \multicolumn{1}{c}{$\beta$} & \multicolumn{1}{c}{$\sigma_{\mathrm{u}}^{2}$} & $\sigma_{\mathrm{e}}^{2}$ & $\gamma$ & \multicolumn{1}{c}{$\mathrm{h}^{2}$} \\
\hline 1 & 0.072 & -0.018 & 1.002 & -0.018 & -0.073 \\
2 & -0.032 & 0.013 & 0.966 & 0.013 & 0.052 \\
3 & 0.040 & 0.029 & 1.013 & 0.028 & 0.110 \\
4 & -0.025 & 0.087 & 1.056 & 0.083 & 0.306 \\
\hline
\end{tabular}

Analysis using the prior for $\sigma_{e}^{2}$ and $\sigma_{u}^{2}$

Bayesian analyses of the four data sets are given using the prior distribution in [2] with $\nu_{e}=\nu_{u}=1, k_{e}=0.975, k_{u}=0.025$ and $g=\frac{1}{3}$. The prior distribution for $\sigma_{e}^{2}$ and $\sigma_{u}^{2}$ is proper and leads to a proper joint posterior distribution (as shown in the Appendix), but the small values chosen for $\nu_{e}$ and $\nu_{u}$ correspond to a very dispersed distribution; this is intended to reveal any problems with convergence of the Gibbs sampler algorithm. The sample data provide little information about $\sigma_{u}^{2}$ as there are only 25 sires. The algorithm is carried out for the four sets of data with the following implementations, which each provide 1000 Gibbs samples:

(a) a single run of 1000 iterations;

(b) taking every (1) 10th, (2) 20th and (3) 30th value in single runs of length 10000 , 20000 and 30000 , respectively, using only one starting value for the parameter vector;

(c) short runs of length (1) 10, (2) 20 and (3) 30, storing the last iterate and replicating this process 1000 times in each case. 
The parameter values used to generate the data were also employed as starting values for all three implementations. No iterates were discarded since the true parameter values were known.

The resulting marginal posterior means and standard deviations for $\beta, \sigma_{u}^{2}, \sigma_{e}^{2}$, $\gamma$ and $h^{2}$ for the four data sets are shown in table II. This table reveals little difference between the implementations in the marginal posterior means, except for a slight tendency for standard deviations to be lower using (a) for $\beta, \sigma_{u}^{2}, \gamma$ and $h^{2}$. Figures 1-4 show the estimated marginal posterior densities for $\beta, \sigma_{u}^{2}, \sigma_{e}^{2}$ and $h^{2}$ using density estimators with normal kernels under the prior specification of this subsection (with implementation (a)) and that considered in the next subsection.

\section{Analysis assuming prior independence of $\sigma_{e}^{2}$ and $\gamma$}

We now compare the above analyses with those based on the second prior specification, which assumes prior independence of $\sigma_{e}^{2}$ and $\gamma$ and prior density [8] for $\gamma$; we use adaptive rejection sampling on the full conditional posterior distribution of $\delta=\ln \gamma$, as defined in the Appendix. The Appendix also describes how the values of $a$ and $b$ in the Beta prior for $\gamma$ are determined from those of $\nu_{e}, \nu_{u}, k_{e}$ and $k_{u}$.

At each Gibbs sampling iteration, adaptive rejection sampling requires at least two points for the construction of the rejection envelope and squeezing function (Gilks and Wild, 1992). The parameter space for $\delta$ is unbounded on the left, so Gilks and Wild (1992) advise that the smallest of these initial points be chosen where the derivative of the log posterior density is positive. The 15th and 85th percentiles of the sampling density from the previous iteration were tried as initial points, additional points being supplied if the derivatives at these values had the same sign. Three evaluations of the $\log$ posterior density of $\delta$ were required on average at each iteration of adaptive rejection sampling.

Convergence of the Gibbs sampler, assessed by monitoring summary statistics for each parameter based on every ten iterations, had clearly occurred before 1000 iterations. The marginal posterior summaries shown in table II and the density estimates for $\beta, \sigma_{u}^{2}, \sigma_{e}^{2}$ and $h^{2}$ in figures 1-4 are based on this number of iterations. The results obtained using the two prior specifications agree very closely, especially those for $\beta$ and $\sigma_{e}^{2}$.

\section{DISCUSSION}

Our results from using Gibbs sampling on a balanced one-way sire model with the prior density of expression [2] for $\sigma_{e}^{2}$ and $\sigma_{u}^{2}$ suggest that it is unnecessary to discard all but the 10 th, 20 th or 30 th iterate; doing so is probably quite wasteful. A much larger proportion of the iterates should be used unless storage is an issue. Using 1000 successive iterates seems adequate to make inferences about the parameters of interest, although the slightly lower standard deviations obtained with implementation (a) may reflect the need to discard 'burn-in' iterations. We encountered no difficulties with the convergence of the Gibbs sampling algorithms used. With real data, we would use REML estimates as starting values for the Gibbs iterations and monitor convergence diagnostics to decide how many 'burnin' iterations to discard (Gilks et al, 1996). 
Table II. Marginal posterior means and standard deviations (SD) of individual parameters for four data sets using 1000 Gibbs samples. Implementations (a), (b1)-(b3) and (c1)-(c3) are based on the prior distribution for $\sigma_{e}^{2}$ and $\sigma_{u}^{2}$, but use different implementations of the Gibbs sampler; (d) assumes prior independence of $\sigma_{e}^{2}$ and $\gamma$ and also uses adaptive rejection sampling.

\begin{tabular}{|c|c|c|c|c|c|c|c|c|c|c|}
\hline & \multicolumn{2}{|c|}{$\beta$} & \multicolumn{2}{|c|}{$\sigma_{\mathrm{u}}^{2}$} & \multicolumn{2}{|c|}{$\sigma_{\mathrm{e}}^{2}$} & \multicolumn{2}{|c|}{$\gamma$} & \multicolumn{2}{|c|}{$\mathrm{h}^{2}$} \\
\hline & Mean & $S D$ & Mean & $S D$ & Mean & $S D$ & Mean & $S D$ & Mean & $S D$ \\
\hline \multicolumn{11}{|c|}{ Data set 1} \\
\hline a & 0.0712 & 0.0489 & 0.0120 & 0.0079 & & 0.0636 & 0.0121 & 0.0079 & 0.0477 & 0.0306 \\
\hline b1 & 0.0724 & 0494 & 0117 & 0.0077 & 79 & & & & & \\
\hline b2 & 0.0722 & 0.0494 & 0117 & .0078 & & & 119 & & & \\
\hline b3 & 0.0719 & & 117 & .0079 & & & & & & \\
\hline c1 & 708 & 1 & & 79 & & & & & & \\
\hline $\mathrm{c} 2$ & 702 & & & & & & & & & \\
\hline c3 & & & & & & & & & & \\
\hline $\mathrm{d}$ & 0.0716 & & & & & & & & & 0.0383 \\
\hline \multicolumn{11}{|c|}{ Data set 2} \\
\hline a & & & & & & & & & & \\
\hline b1 & -0.0 & & & & & & & & & \\
\hline b2 & -0.0318 & 0.0525 & & & & & & & & 571 \\
\hline b3 & -0.0325 & 0.0538 & 0.0202 & 0.0145 & & & & & & 578 \\
\hline $\mathrm{cl}$ & -0.0344 & 0.0522 & & 0.0144 & & & & & & 572 \\
\hline c2 & -0.0321 & 0.0516 & & & & & & & & 582 \\
\hline c3 & -0.0 & 0.0515 & & & & & & & & \\
\hline $\mathrm{d}$ & -0.0311 & 0.0500 & 0.0182 & 0.0146 & 0.9677 & & & & & 0.0568 \\
\hline \multicolumn{11}{|c|}{ Data set 3} \\
\hline a & & & & & & & & & & 1.0695 \\
\hline b1 & & & & & & & & & & \\
\hline $\mathrm{b} 2$ & & 0.0569 & & & & & & & & 0.0729 \\
\hline b3 & & 0.0576 & & & & & & & & 0.0742 \\
\hline $\mathrm{c} 1$ & & 0.0573 & & & & & & & & 0.0792 \\
\hline $\mathrm{c} 2$ & & 0.0565 & & & & & & & & 0.0708 \\
\hline c3 & & & & 0.0202 & & & & & & 0.0744 \\
\hline d & 0.0407 & 0.0558 & 0.0288 & & 1.0204 & 0.0660 & & & & 0.0797 \\
\hline \multicolumn{11}{|c|}{ Data set 4} \\
\hline a & -0.0272 & 0.0715 & 0.0861 & 0.0407 & & 0.0704 & & & & \\
\hline b1 & & 0.0777 & & 0.0426 & & 0.0694 & & & & \\
\hline $\mathrm{b} 2$ & -0.0257 & 0.0768 & 0.0847 & 0.0430 & & 0.0666 & & & & 0.1357 \\
\hline b3 & -0.0237 & 0.0781 & 0.0828 & 0.0406 & 1.0651 & & & & & 0.1298 \\
\hline $\mathrm{c} 1$ & -0.0217 & 0.0713 & 0.0826 & 0.0413 & 1.9661 & & & & 0.2851 & 0.1335 \\
\hline c2 & -0.0223 & 0.0734 & 0.0822 & 0.0410 & & & & & 0.2839 & 0.1321 \\
\hline c3 & -0.0257 & 0.0763 & 0.0850 & 0.0431 & & & & & & 0.1382 \\
\hline d & -0.0261 & 0.0731 & 0.0887 & 0.0388 & 1.0632 & 0.0686 & 0.0837 & 0.0362 & 0.3047 & 0.1209 \\
\hline
\end{tabular}




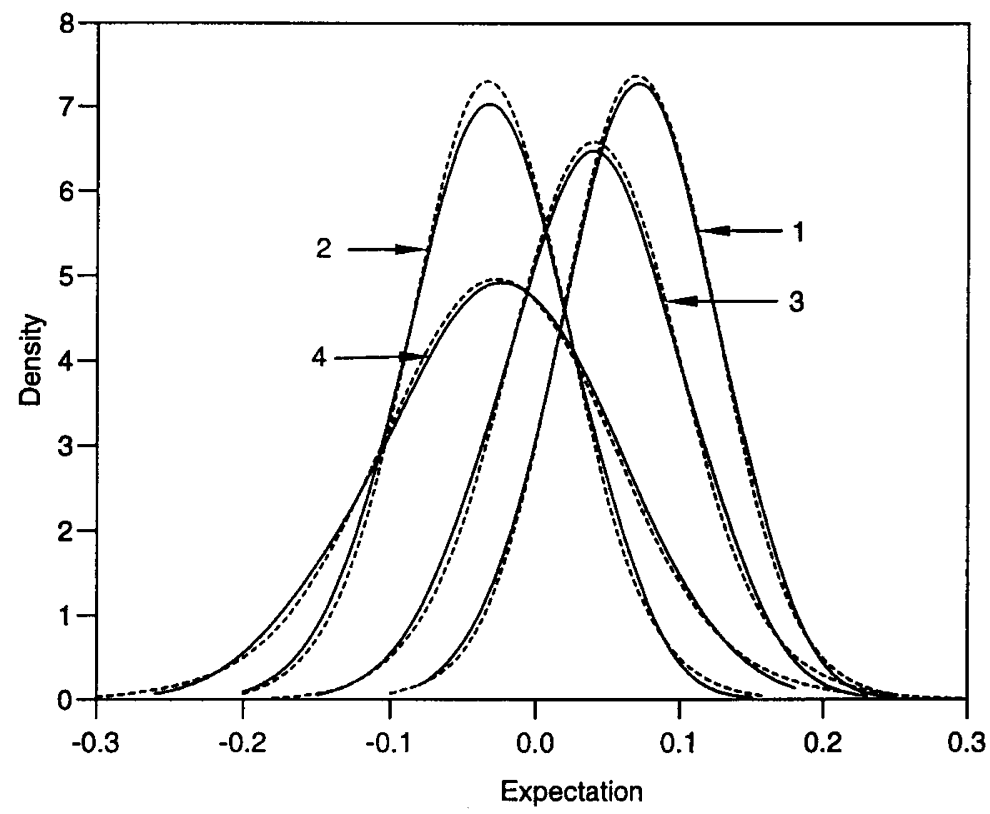

Fig 1. Estimated marginal posterior densities of $\beta$ for four sets of data; - , prior specification for $\sigma_{e}^{2}$ and $\sigma_{u}^{2}$; - - - - prior specification for $\sigma_{e}^{2}$ and $\gamma$.

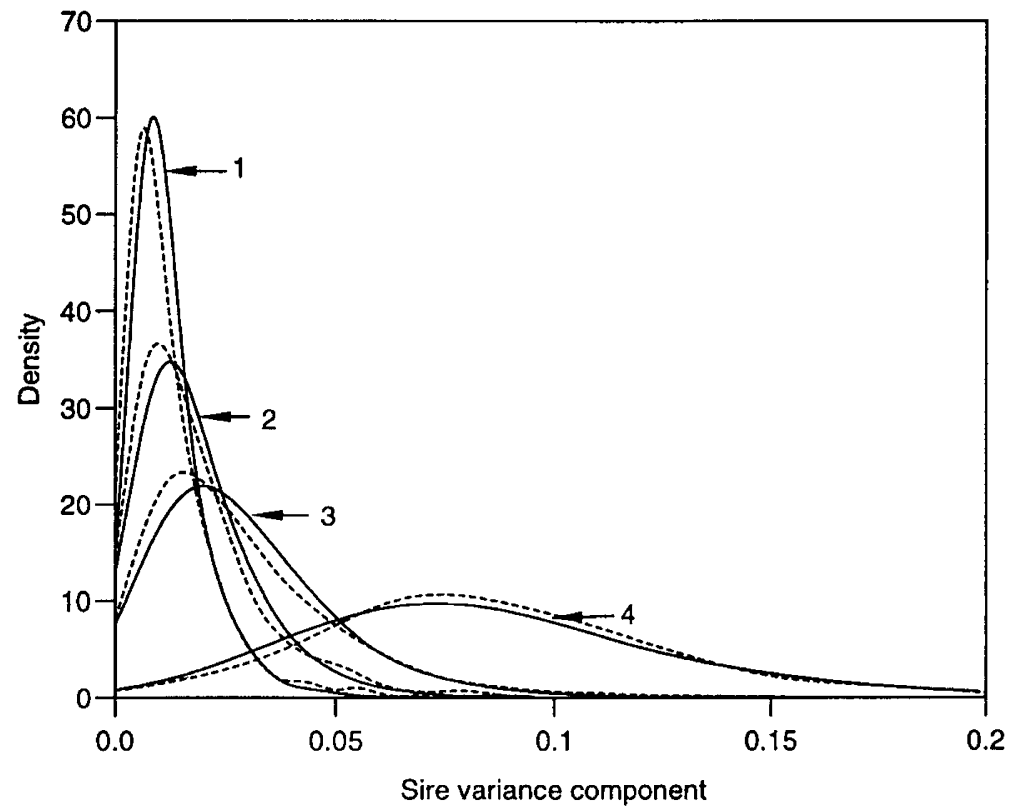

Fig 2. Estimated marginal posterior densities of $\sigma_{u}^{2}$ for four sets of data; -, prior specification for $\sigma_{e}^{2}$ and $\sigma_{u}^{2} ;-\cdots$, , prior specification for $\sigma_{e}^{2}$ and $\gamma$. 


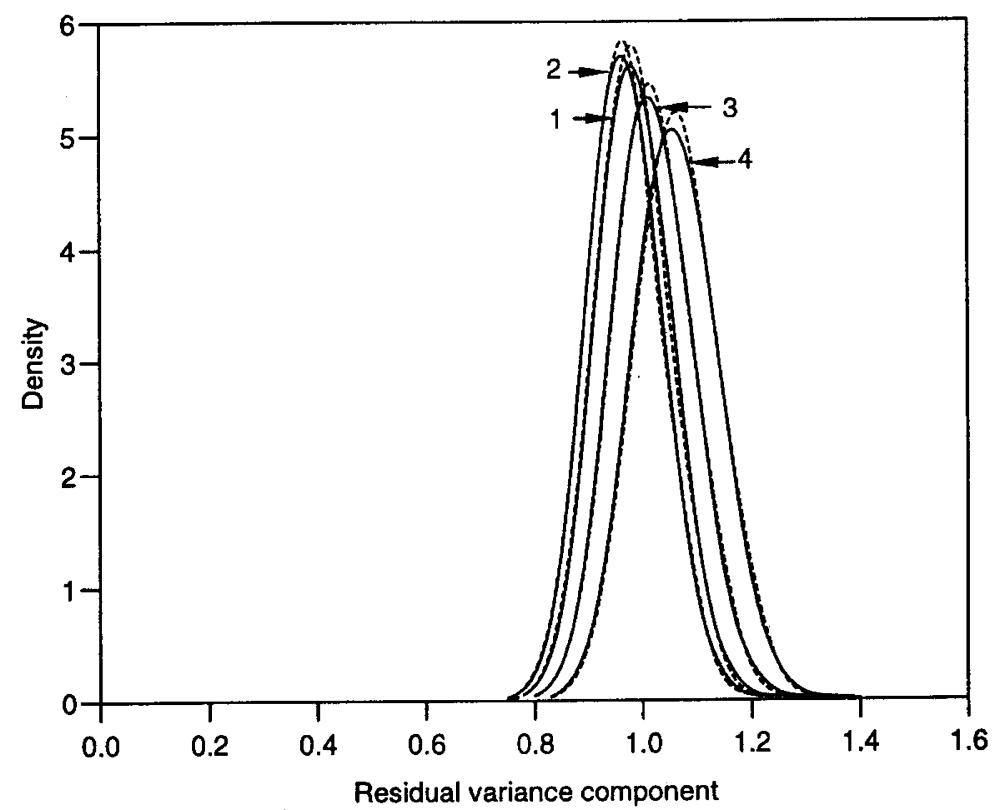

Fig 3. Estimated marginal posterior densities of $\sigma_{e}^{2}$ for four sets of data; -, prior specification for $\sigma_{e}^{2}$ and $\sigma_{u}^{2}$; - - , prior specification for $\sigma_{e}^{2}$ and $\gamma$.

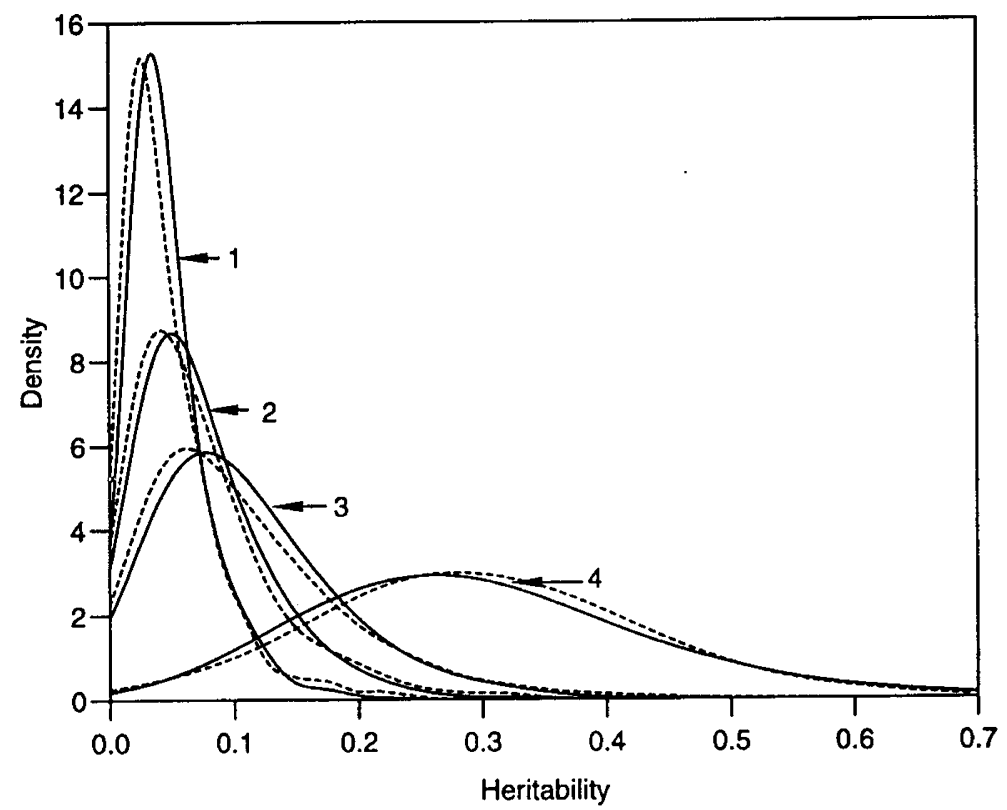

Fig 4. Estimated marginal posterior densities of $h^{2}$ for four sets of data; 一, prior specification for $\sigma_{e}^{2}$ and $\sigma_{u}^{2} ;-\cdots$, , prior specification for $\sigma_{e}^{2}$ and $\gamma$. 
The prior distribution which assumes $\sigma_{e}^{2}$ and $\gamma$ to be independent provides a potentially useful alternative to the usual prior in which $\sigma_{e}^{2}$ and $\sigma_{u}^{2}$ are taken to be independent. The adaptive rejection sampling algorithm of Gilks and Wild (1992) is found to work efficiently with the resulting full conditional distribution for $\ln \gamma$.

The sensitivity of a Bayesian analysis to the choice of prior hyperparameters and to different functional forms of prior can be investigated. For our simulated data, the use of an alternative form of prior distribution led to very similar marginal posterior inferences on each parameter.

The use of the alternative prior distribution for $\sigma_{e}^{2}$ and $\gamma$ can be extended to models with further variance components, for example if [1] is generalized to

$$
\mathbf{y}=\mathbf{X} \boldsymbol{\beta}+\mathbf{Z u}+\mathbf{T} \mathbf{v}+\mathbf{e}
$$

where $\mathbf{v}$ is an $r$-vector of random effects, $\mathbf{T}$ is a known $n \times r$ matrix and $\mathbf{v}$ is taken to be $N_{r}\left(\mathbf{0}, \sigma_{v}^{2} \mathbf{H}\right)$ independently of $\boldsymbol{\beta}, \mathbf{u}$ and $\mathbf{e}$ with $\mathbf{H}$ a known matrix. If $\boldsymbol{\beta}, \sigma_{e}^{2}$ and $\sigma_{v}^{2}$ are taken to be mutually independent a priori, with uniform, $\chi^{-2}\left(\nu_{e},\left(\nu_{e} k_{e}\right)^{-1}\right)$ and $\chi^{-2}\left(\nu_{v},\left(\nu_{v} k_{v}\right)^{-1}\right)$ distributions, respectively, and $\gamma$ is independent of them with the Beta distribution defined in [8], then the full conditional distributions for $\boldsymbol{\beta}, \mathbf{u}, \mathbf{v}, \sigma_{e}^{2}$ and $\sigma_{v}^{2}$ are, respectively,

$$
\begin{gathered}
N_{p}\left(\left(\mathbf{X}^{\prime} \mathbf{X}\right)^{-1} \mathbf{X}^{\prime}(\mathbf{y}-\mathbf{Z} \mathbf{u}-\mathbf{T} \mathbf{v}), \sigma_{e}^{2}\left(\mathbf{X}^{\prime} \mathbf{X}\right)^{-1}\right) \\
N_{q}\left(\left(\mathbf{Z}^{\prime} \mathbf{Z}+\gamma^{-1} \mathbf{G}^{-1}\right)^{-1} \mathbf{Z}^{\prime}(\mathbf{y}-\mathbf{X} \boldsymbol{\beta}-\mathbf{T} \mathbf{v}), \sigma_{e}^{2}\left(\mathbf{Z}^{\prime} \mathbf{Z}+\gamma^{-1} \mathbf{G}^{-1}\right)^{-1}\right) \\
N_{r}\left(\left(\mathbf{T}^{\prime} \mathbf{T}+\varepsilon^{-1} \mathbf{H}^{-1}\right)^{-1} \mathbf{T}^{\prime}(\mathbf{y}-\mathbf{X} \boldsymbol{\beta}-\mathbf{Z u}), \sigma_{e}^{2}\left(\mathbf{T}^{\prime} \mathbf{T}+\varepsilon^{-1} \mathbf{H}^{-1}\right)^{-1}\right) \\
\chi^{-2}\left(n+q+\nu_{e},\left[(\mathbf{y}-\mathbf{X} \boldsymbol{\beta}-\mathbf{Z u}-\mathbf{T} \mathbf{v})^{\prime}(\mathbf{y}-\mathbf{X} \boldsymbol{\beta}-\mathbf{Z u}-\mathbf{T} \mathbf{v})\right.\right. \\
\left.\left.+\nu_{e} k_{e}+\gamma^{-1} \mathbf{u}^{\prime} \mathbf{G}^{-1} \mathbf{u}\right]^{-1}\right)
\end{gathered}
$$

and

$$
=\chi^{-2}\left(r+\nu_{v},\left[\mathbf{v}^{\prime} \mathbf{H}^{-1} \mathbf{v}+\nu_{v} k_{v}\right]^{-1}\right)
$$

where $\varepsilon$ denotes $\sigma_{v}^{2} / \sigma_{e}^{2}$, while that for $\gamma$ is the same as [11].

\section{ACKNOWLEDGEMENTS}

The authors are grateful to W Gilks for providing a FORTRAN program to perform adaptive rejection sampling. MZ Firat was supported by the Turkish Government.

\section{REFERENCES}

Cantet RJC, Fernando RL, Gianola D (1992) Bayesian inference about dispersion parameters of univariate mixed models with maternal effects: theoretical considerations. Genet Sel Evol 24, 107-135

Devroye L (1986) Non-Uniform Random Variate Generation. Springer-Verlag, New York

Foulley JL, Im S, Gianola D, Hoschele I (1987) Empirical Bayes estimation of parameters for $n$ polygenic binary traits. Genet Sel Evol 19, 197-224 
Gelfand AE, Smith AFM (1990) Sampling-based approaches to calculating marginal densities. J Am Stat Assoc 85, 398-409

Gelfand AE, Hills SE, Racine-Poon A, Smith AFM (1990) Illustration of Bayesian inference in normal data models using Gibbs sampling. J Am Stat Assoc 85, 972-985

Gelman, A, Rubin DB (1992) A single series from the Gibbs sampler provides a false sense of security. In: Bayesian Statistics 4 (JM Bernardo, JO Berger, AP David, AFM Smith, eds), Clarendon Press, Oxford, 625-631

Geman S, Geman D (1984) Stochastic relaxation, Gibbs distributions and the Bayesian restoration of images. IEEE Transactions on Pattern Analysis and Machine Intelligence $6,721-741$

Gianola D, Fernando RL (1986) Bayesian methods in animal breeding theory. J Anim Sci $63,217-244$

Gianola D, Foulley JL (1990) Variance estimation from integrated likelihoods (VEIL). Genet Sel Evol 22, 403-417

Gianola D, Foulley JL, Fernando RL (1986) Prediction of breeding values when variances are not known. Genet Sel Evol 18, 485-498

Gilks WR, Richardson S, Spiegelhalter DJ (1996) Markov Chain Monte Carlo in Practice. Chapman and Hall, London

Gilks WR, Wild P (1992) Adaptive rejection sampling for Gibbs sampling. Appl Statist 41, 337-348

Harville DA (1977) Maximum likelihood approaches to variance component estimation and to related problems. J Am Stat Assoc 72, 320-338

Patterson HD, Thompson R (1971) Recovery of inter-block information when block sizes are unequal. Biometrika 58, 545-554

Raftery AE, Lewis SM (1992) How many iterations in the Gibbs sampler? In: Bayesian Statistics 4 (JM Bernardo, JO Berger, AP David, AFM Smith, eds), Clarendon Press, Oxford, 763-773

Silverman BW (1986) Density Estimation for Statistics and Data Analysis. Chapman and Hall, London

Wang CS, Rutledge JJ, Gianola, D (1993) Marginal inferences about variance components in a mixed linear model using Gibbs sampling. Genet Sel Evol 25, 41-62

Wang CS, Rutledge JJ, Gianola D (1994) Bayesian analysis of mixed linear models via Gibbs sampling with an application to litter size in Iberian pigs. Genet Sel Evol 26, 91-115

\section{APPENDIX}

Marginal posterior probability density function of $\gamma$ using the prior for $\sigma_{e}^{2}$ and $\sigma_{u}^{2}$

Integrating the posterior density for $\boldsymbol{\beta}, \mathbf{u}, \sigma_{e}^{2}$ and $\sigma_{u}^{2}$ given in [3] with respect to $\boldsymbol{\beta}$ and $\mathbf{u}$, the posterior density of $\sigma_{e}^{2}$ and $\sigma_{u}^{2}$ is found to be proportional to

$$
\left(\sigma_{e}^{2}\right)^{-\frac{1}{2}\left(n+\nu_{e}-p-q+2\right)}\left(\sigma_{u}^{2}\right)^{-\frac{1}{2}\left(q+\nu_{u}+2\right)}\left|\mathbf{Z}^{\prime} \mathbf{Q Z}+\gamma^{-1} \mathbf{G}^{-1}\right|^{-\frac{1}{2}} \exp \left[-\sigma_{e}^{-2} D(\gamma)\right]
$$

where $\mathbf{Q}$ denotes $\mathbf{I}_{n}-\mathbf{X}\left(\mathbf{X}^{\prime} \mathbf{X}\right)^{-1} \mathbf{X}^{\prime}$ and

$$
D(\gamma)=\frac{1}{2}\left\{\nu_{e} k_{e}+\gamma^{-1} \nu_{u} k_{u}+\mathbf{y}^{\prime}\left[\mathbf{Q}-\mathbf{Q Z}\left(\mathbf{Z}^{\prime} \mathbf{Q Z}+\gamma^{-1} \mathbf{G}^{-1}\right)^{-1} \mathbf{Z}^{\prime} \mathbf{Q}\right] \mathbf{y}\right\}
$$


The joint density of $\sigma_{e}^{2}$ and $\gamma$ is therefore proportional to

$$
\left(\sigma_{e}^{2}\right)^{-\frac{1}{2}\left(n+\nu_{e}+\nu_{u}-p+2\right)} \gamma^{-\frac{1}{2}\left(\nu_{u}+2\right)}\left|\mathbf{G}^{-1}+\gamma \mathbf{Z}^{\prime} \mathbf{Q Z}\right|^{-\frac{1}{2}} \exp \left[-\sigma_{e}^{-2} D(\gamma)\right]
$$

and the marginal posterior density of $\gamma$ is proportional to

$$
\gamma^{-\frac{1}{2}\left(\nu_{u}+2\right)}\left|\mathbf{G}^{-1}+\gamma \mathbf{Z}^{\prime} \mathbf{Q Z}\right|^{-\frac{1}{2}}[D(\gamma)]^{-\frac{1}{2}\left(n+\nu_{e}+\nu_{u}-p\right)} \quad(0<\gamma<g)
$$

provided that $n+\nu_{e}+\nu_{u}-p$ is positive. Whether or not this is a proper density function depends on its behaviour near zero. For small $\gamma,\left|\mathbf{G}^{-1}+\gamma \mathbf{Z}^{\prime} \mathbf{Q Z}\right|$ is approximately constant, while the $\gamma^{-1} \nu_{u} k_{u}$ term is dominant in $D(\gamma)$ so long as $\nu_{u}$ is positive. So the density of $\gamma$ behaves like $\gamma^{\frac{1}{2}\left(n+\nu_{e}-p-2\right)}$ near zero, and has a finite integral if the exponent exceeds -1 , that is if $n+\nu_{e}$ exceeds $p$.

\section{Matching quartiles of $\gamma$ in the two prior distributions}

To compare inferences under the two prior distributions considered in this article, we determine values of the hyperparameters $a$ and $b$ defining the Beta distribution for $\gamma$ in the second prior so that the two specifications are matched in some sense. The first specification for the variance parameters (in terms of $\sigma_{e}^{2}$ and $\sigma_{u}^{2}$ ) implies that $\gamma$ (which equals $\sigma_{u}^{2} / \sigma_{e}^{2}$ ) has probability density function proportional to

$$
\gamma^{\frac{1}{2}\left(\nu_{e}-2\right)}\left(1+\frac{\nu_{e} k_{e}}{\nu_{u} k_{u}} \gamma\right)^{-\frac{1}{2}\left(\nu_{u}+\nu_{e}\right)} \quad(0<\gamma<g)
$$

Hence $\phi=\gamma k_{e} / k_{u}$ has an $F\left(\nu_{e}, \nu_{u}\right)$ distribution truncated at $g k_{e} / k_{u}$. We choose $a$ and $b$ to match the Beta distribution used for $\gamma$ in the second prior to this by equating their quartiles. If $G$ denotes the distribution function of $F\left(\nu_{e}, \nu_{u}\right)$ then $\phi$ has lower and upper quartiles $\phi_{\mathrm{L}}, \phi_{\mathrm{U}}$ which are solutions of

$$
G\left(\phi_{\mathrm{L}}\right)=0.25 G\left(g k_{e} / k_{u}\right), \quad G\left(\phi_{\mathrm{U}}\right)=0.75 G\left(g k_{e} / k_{u}\right)
$$

and $\gamma$ has quartiles $\phi_{\mathrm{L}} k_{e} / k_{u}$ and $\phi_{\mathrm{U}} k_{e} / k_{u}$. Thus if $x_{\mathrm{L}}$ and $x_{\mathrm{U}}$ denote the lower and upper quartiles of the Beta distribution $B(a, b)$, we equate $x_{\mathrm{L}}$ and $x_{\mathrm{U}}$ to $\phi_{\mathrm{L}} k_{u} /\left(g k_{e}\right)$ and $\phi_{\mathrm{U}} k_{u} /\left(g k_{e}\right)$, respectively, by solving for $a$ and $b$ the two equations

$$
\frac{1}{B(a, b)} \int_{0}^{x_{\mathrm{L}}} x^{a-1}(1-x)^{b-1} \mathrm{~d} x=0.25, \frac{1}{B(a, b)} \int_{0}^{x_{\mathrm{U}}} x^{a-1}(1-x)^{b-1} \mathrm{~d} x=0.75
$$

Example: We use the prior specification for $\sigma_{e}^{2}$ and $\sigma_{u}^{2}$ with $\nu_{e}=\nu_{u}=1$, $k_{e}=0.975, k_{u}=0.025$ and $g=\frac{1}{3}$, so that $\gamma k_{e} / k_{u}$ has an $F(1,1)$ distribution truncated at $\frac{1}{3} k_{e} / k_{u}=13 ; G(13)=0.8278$, so

$$
\begin{aligned}
\phi_{\mathrm{L}}=G^{-1}(0.25 \times 0.8278) & =0.1136, \quad \phi_{\mathrm{U}}=G^{-1}(0.75 \times 0.8278)=2.1777 \\
x_{\mathrm{L}}=0.1136 / 13 & =0.0087, \quad x_{\mathrm{U}}=2.1777 / 13=0.1675
\end{aligned}
$$


The corresponding values of $a$ and $b$ are 0.4038 and 3.0678 .

\section{Log-concavity of the full conditional distributions of $\gamma$ and $\ln \gamma$}

A density is defined to be log-concave if the derivative of its logarithm is monotone decreasing. For the density of $\gamma$ in [11], the required derivative is

$$
\frac{1}{2} \frac{2 a-2-q}{\gamma}-\frac{b-1}{g-\gamma}+\frac{1}{2} \frac{\mathbf{u}^{\prime} \mathbf{G}^{-1} \mathbf{u}}{\gamma^{2} \sigma_{e}^{2}} \quad(0<\gamma<g)
$$

The second and third terms are decreasing in $\gamma$ if $b$ exceeds 1 , but the first is not unless $a$ is greater than $\frac{1}{2} q+1$; log-concavity cannot be guaranteed for all $\sigma_{e}^{2}$ without this condition.

If we transform to $\delta=\ln \gamma$ (and include the Jacobian of the transformation), the logarithm of the density is, apart from an additive constant,

$$
\left(a-\frac{1}{2} q\right) \delta+(b-1) \ln \left(g-e^{\delta}\right)-\frac{1}{2} \sigma_{e}^{-2} e^{-\delta} \mathbf{u}^{\prime} \mathbf{G}^{-1} \mathbf{u} \quad(-\infty<\delta<\ln g)
$$

and this has derivative

$$
\left(a-\frac{1}{2} q\right)-\frac{b-1}{g-e^{\delta}}+\frac{1}{2} \sigma_{e}^{-2} e^{-\delta} \mathbf{u}^{\prime} \mathbf{G}^{-1} \mathbf{u} \quad(-\infty<\delta<\ln g)
$$

The first term is constant and the second and third are decreasing with respect to $\delta$ (so that the density is log-concave) as long as $b$ is at least 1. 